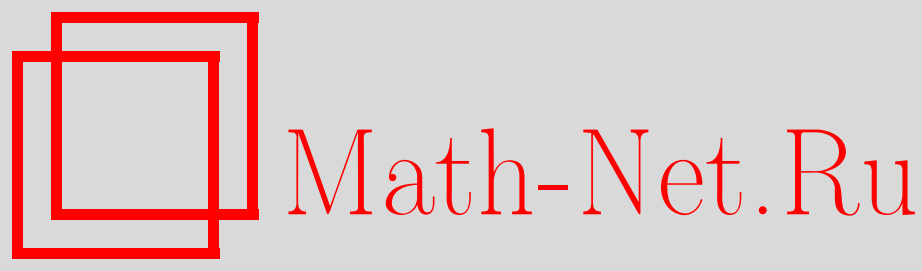

А. Москосо, К. Андрес, К. Пахарес, Высокоэнергетический предел КХД и новые экспериментальные данные Большого андронного коллайдера, ТМФ, 2013, том 176, номер 1, 127-139

DOI: https://doi.org/10.4213/tmf8493

Использование Общероссийского математического портала Math-Net.Ru подразумевает, что вы прочитали и согласны с пользовательским соглашением http://www. mathnet.ru/rus/agreement

Параметры загрузки:

IP : 54.174 .149 .18

26 апреля 2023 г., $11: 53: 43$

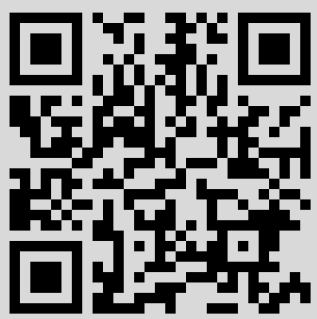




\title{
ВЫСОКОЭНЕРГЕТИЧЕСКИЙ ПРЕДЕЛ КХД И НОВЫЕ ЭКСПЕРИМЕНТАЛЬНЫЕ ДАННЫЕ БОЛЬШОГО АНДРОННОГО КОЛЛАЙДЕРА
}

\begin{abstract}
Анализируются некоторые следствия экспериментальных данных, полученных на детекторах RHIC и Большого адронного коллайдера и касающихся материи с высокой плотностью, особое внимание уделяется разнице между Большим Взрывом и малым большим взрывом. Обсуждаются распределения быстрот производимых частиц, геометрическое масштабирование, насыщающий импульс глюонов, гармонические моменты и дальнодействующие корелляции быстрот.
\end{abstract}

Ключевые слова: тяжелые ионы, геометрическое масштабирование, малый большой взрыв.

DOI: $10.4213 / \operatorname{tmf} 8493$

\section{1. ВВЕДЕНИЕ}

Новые данные, полученные в последние два года на детекторах ALICE, CMS и ATLAS, в совокупности с предыдущими данными, полученными на RHIC и SPS, изменили наши взгляды на поведение КХД при высокой плотности и/или высокой температуре. В настоящей работе дается обзор последних достижений в данной области, особое внимание уделяется сходствам и различиям между материями с высокой плотностью, образующимися при Большом Взрыве и при характерных энергиях на Большом адронном коллайдере (БАК), последние условия часто называются малым большим взрывом.

Сначала опишем общие характеристики малого большого взрыва с учетом соответствующих общих характеристик Большого Взрыва, таких как возраст и плотность массы. Для определения наблюдаемой плотности энергии необходимо знать распределение быстрот порождаемых частиц при АА-соударениях с заданными энергиями и прицельным параметром. Мы проанализируем последние данные БАК, которые касаются насыщающего импульса глюонов $Q_{\mathrm{s}}$. Величина $Q_{\mathrm{s}}$ естественным образом

* Departamento de Física de Particulas и IGFAE, Universidade de Santiago de Compostela, Santiago de Compostela, Spain.

E-mail: alexis.moscoso@rai.usc.es, carlota.andres.casas@gmail.com, pajares@fpaxp1.usc.es 
определяет новый масштаб КХД. На основе данных о распределении поперечных импульсов мы также обсудим геометрическое масштабирование. Нуклеосинтез Н, $\mathrm{d}, \mathrm{He}$ и Li в условиях Большого Взрыва на временах $t \sim 100$ с приводит к нехватке различных элементарных частиц $(\pi, \mathrm{K}, \mathrm{p}, \Lambda, \Sigma, \ldots)$ при малом большом взрыве после установления режима размораживания. На этом этапе, по прошествии примерно $t \sim 10^{-23}$ с после соударения, адроны отделяются друг от друга. Структуры, формирующиеся при соударении и основанные на конфигурациях кварковых и глюонных полей (кластеры, струны, джеты и т. п.), эволюционируют и распадаются на адроны. Этот процесс частично отвечает хаббловскому расширению. Флуктуации плотности приводят к образованию крупномасштабных структур Вселенной, и изучение флуктуаций последовательности событий позволяет исследовать фазовые переходы между связанными в адроны кварками и глюонами и свободными кварками и глюонами. Микроволновое фоновое излучение, производимое отщеплением фотонов от материи, аналогично испусканию тепловых фотонов при малом поперечном импульсе из вещества, образуемого тепловыми кварками и глюонами. Наконец, так же, как флуктуации температуры предоставляют информацию о самых ранних стадиях эпохи Большого Взрыва, можно получить информацию о ранних стадиях соударения, исследуя дальние корреляции быстрот, а также так называемую хребтовую структуру, наблюдаемую при энергиях RHIC и БАК в высокомультипликативных рр-событиях и в $\mathrm{pPb}$ - и АА-соударениях.

Ввиду ограниченности объема статьи невозможно обсудить все перечисленные выше вопросы, так что мы сосредоточимся только на некоторых из них.

\section{2. ГЛОБАЛЬНЫЕ ХАРАКТЕРИСТИКИ МАЛОГО БОЛЬШОГО ВЗРЫВА}

Объем и время между соударением и разморозкой, а именно расщеплением адронов, можно определить с помощью интерферометрии идентичных частиц. Корреляции Бозе-Эйнштейна позволяют измерять различные радиусы $R_{\text {side }}, R_{\text {out }}, R_{\text {long }}$, равно как и время жизни источника. На рис. 1 представлено произведение радиусов (объем источника) как функция распределения мультипликативных псевдобыстрот при различных энергиях. Самая правая точка отвечает центральным $\mathrm{PbPb-соударениям} \mathrm{на} \mathrm{БАК} \mathrm{[1].} \mathrm{Значение} \mathrm{объема} \mathrm{в} \mathrm{этой} \mathrm{точке} \mathrm{в} \mathrm{два} \mathrm{раза} \mathrm{больше,}$ чем для энергий RHIC; его абсолютное значение равно 300 фм³. На рис. 2 показаны измеренные значения времени жизни как функции от кубического корня из распределения мультипликативных псевдобыстрот. Измерения на детекторе ALICE дают результат 10 фм $/ c$, или $3 \cdot 10^{-23}$ с, что в $10^{40}$ раз меньше времени жизни Вселенной, поэтому малый взрыв одновременно является и быстрым взрывом. С другой стороны, из мультипликативности БАК и среднего поперечного импульса можно получить плотности энергии, достигаемые на БАК. Для центральных $\mathrm{PbPb}$-соударений ее значение достигает 10-12 ГэВ $/$ фм $^{3}$, что значительно больше критической плотности энергии фазового перехода. Из различных отношений частиц можно определить достигаемую температуру, которая на $50 \%$ превышает критическую температуру решетки $T_{\mathrm{c}} \sim 160 \mathrm{MэB.}$ 


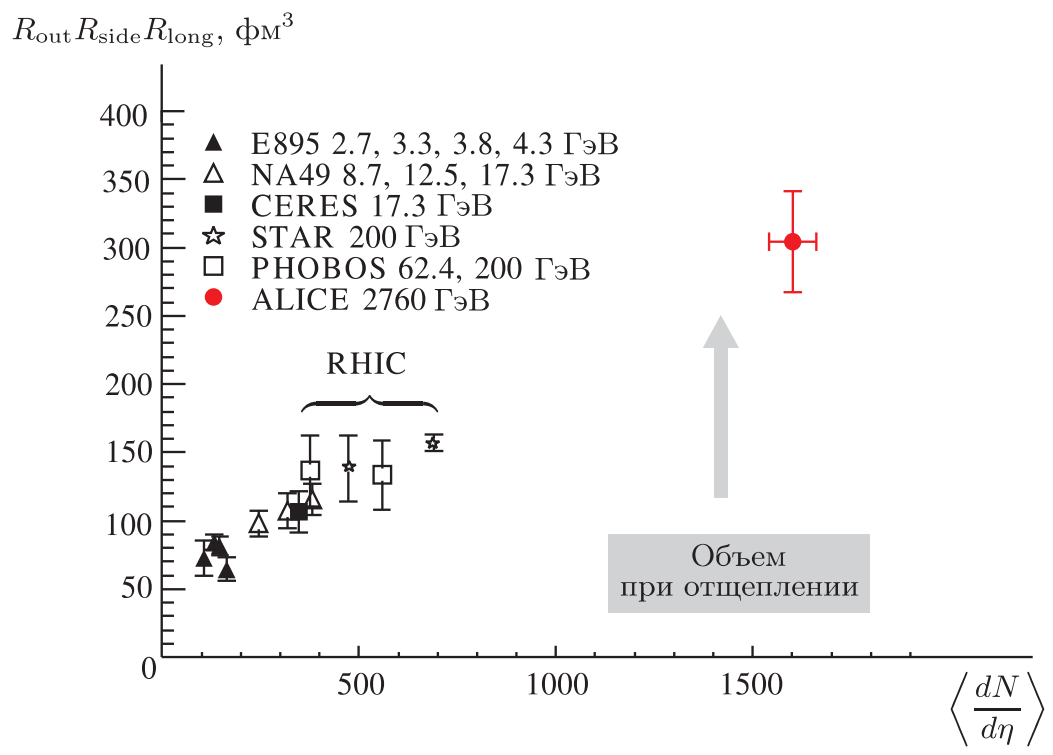

Рис. 1. Произведение радиусов $R_{\text {side }} R_{\text {out }} R_{\text {long }}$ как функция от $d N / d \eta$.

\section{3. РАСПРЕДЕЛЕНИЯ МУЛЬТИПЛИКАТИВНЫХ БЫСТРОТ И НАСЫЩЕНИЕ ГЛЮОНОВ}

Ясно, что число глюонов, задаваемое структурной функцией $x G^{A}\left(x, Q^{2}\right)$, умноженное на поперечный размер отдельного глюона $\alpha_{\mathrm{s}}\left(Q^{2}\right) / Q^{2}$, не может превышать поперечную поверхность ядра $\pi R_{\mathrm{A}}^{2}$. Если аппроксимировать структурную функцию глюонов в ядре выражением $x G^{\mathrm{A}}\left(x, Q^{2}\right) \approx A x G^{\mathrm{N}}\left(x, Q^{2}\right)$, где $x G^{\mathrm{N}}\left(x, Q^{2}\right)$ - структурная функция нуклона, то получим предел насыщения [2], [3]

$$
\frac{\alpha_{\mathrm{s}}\left(Q_{\mathrm{s}}^{2}\right) A x G^{\mathrm{N}}\left(x, Q_{\mathrm{s}}^{2}\right)}{Q_{\mathrm{s}}^{2} \pi R_{\mathrm{A}}^{2}} \approx 1
$$

определяющий импульс насыщения $Q_{\mathrm{s}}$. При $x G^{\mathrm{N}}\left(x, Q_{\mathrm{s}}^{2}\right) \sim 1 / x^{\lambda}$ получаем

$$
Q_{\mathrm{s}}^{2} \sim \frac{A^{1 / 3}}{x^{\lambda}}=s^{\lambda} A^{1 / 3} .
$$

Можно произвести более детальное исследование с помощью эволюционных уравнений. Рассмотрим рассеяние диполя q $\bar{q}$ на адроне. Эволюция диполя с энергией $d Y$, испускающего глюон в результате взаимодействия, задается уравнением Балитского-Фадина-Кураева-Липатова [4], [5] (см. рис. 3)

$$
\frac{\partial N_{x y}}{\partial \tau}=\bar{\alpha}_{\mathrm{s}} \int \frac{d^{2} z_{\mathrm{t}}}{2 \pi} \frac{\left(x_{\mathrm{t}}-y_{\mathrm{t}}\right)^{2}}{\left(x_{\mathrm{t}}-z_{\mathrm{t}}\right)^{2}\left(y_{\mathrm{t}}-z_{\mathrm{t}}\right)^{2}}\left(N_{x z}+N_{z y}-N_{x y}\right),
$$

которое описывает эволюцию амплитуды рассеяния диполя, локализованного в точках $x$ и $y$ с разностью между быстротами адрона и виртуального фотона $(\tau \equiv$ $\left.\ln (1 / x)=y_{\mathrm{h}}-y_{\gamma^{*}}\right)$, как функцию от двух новых рожденных диполей. В уравнении (1) имеем $\bar{\alpha}_{\mathrm{s}}=\alpha_{\mathrm{s}} N_{\mathrm{c}} / \pi$, и поперечный размер диполя $r_{\mathrm{t}}$ задается формулой

5 Теоретическая и математическая физика, т. 176, № 1, 2013 г. 


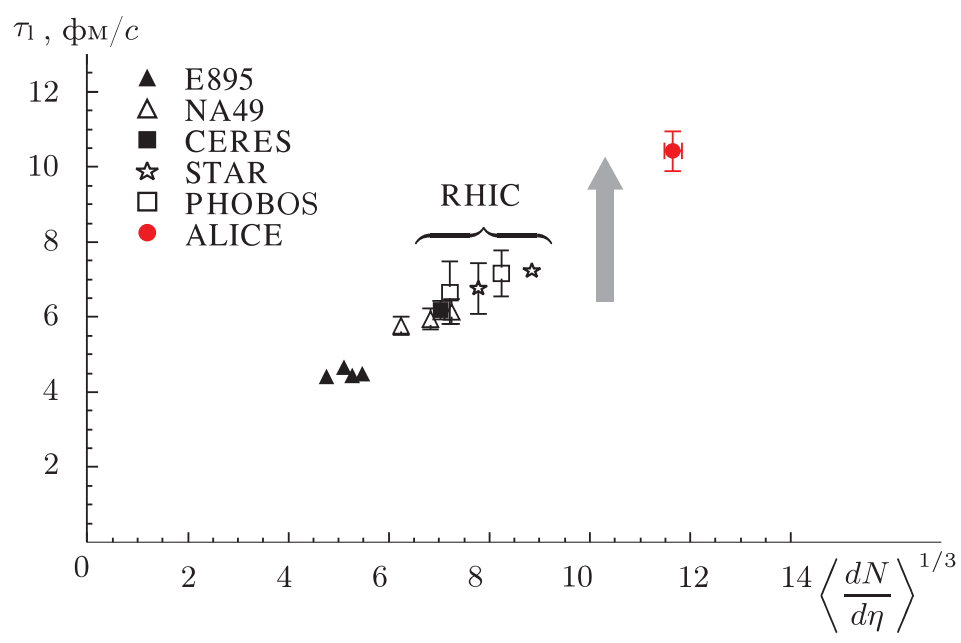

Рис. 2. Время жизни как функция от $(d N / d \eta)^{1 / 3}$.

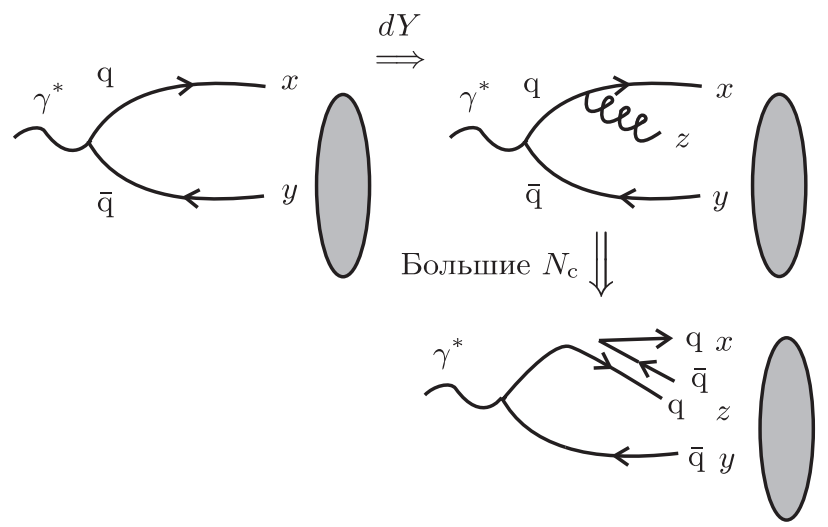

Рис. 3. Представление уравнения Балитского-Фадина-Кураева-Липатова.

$r_{\mathrm{t}}=x_{\mathrm{t}}-y_{\mathrm{t}}$. Решение уравнения (1) имеет вид

$$
N_{\tau}\left(r_{\mathrm{t}}\right)=\sqrt{r_{\mathrm{t}}^{2} Q_{0}^{2}} \frac{e^{\tau \omega \overline{\alpha_{\mathrm{s}}}}}{\sqrt{2 \pi \beta \overline{\alpha_{\mathrm{s}}} \tau}} \exp \left(-\frac{\ln ^{2}\left(r_{\mathrm{t}}^{2} Q_{0}^{2}\right)}{2 \beta \overline{\alpha_{\mathrm{s}}} \tau}\right)
$$

где $\omega$ и $\beta$ - положительные константы. Это решение содержит, к сожалению, два существенных недостатка, а именно:

а) нарушение унитарности, поскольку решение растет как степень энергии.

б) в силу диффузионного поведения глюоны могут переходить в непертурбативную область $k_{\mathrm{t}}^{2}=1 / r_{\mathrm{t}}^{2}<\Lambda_{\mathrm{QCD}}^{2}$.

Эти две проблемы удается разрешить в уравнении Балитского-Ковчегова [6], [7], которое добавляет в уравнение (3) член $N_{x z} N_{z y}$ с отрицательным знаком, описыва- 


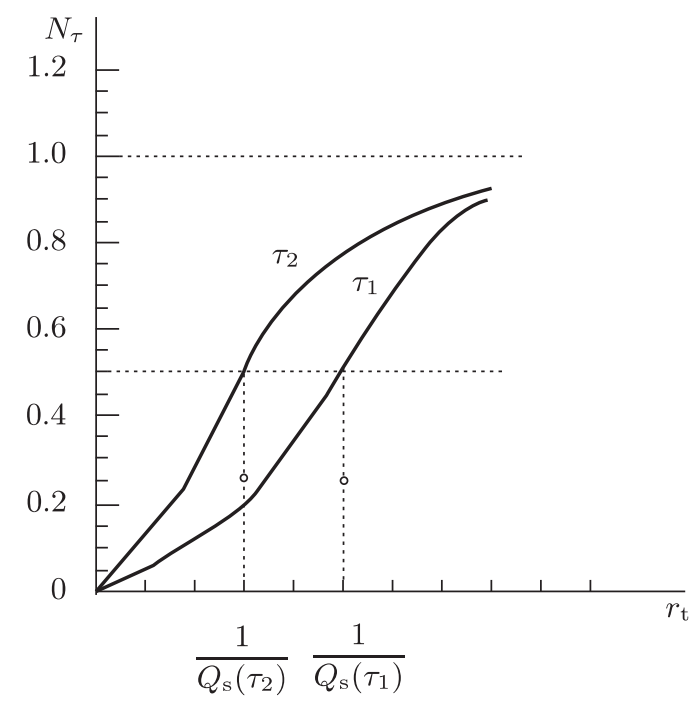

Рис. 4. Амплитудное решение уравнения Балитского-Ковчегова при двух энергиях $\tau_{1}<\tau_{2}$ как функция от размера $r_{\mathrm{t}}$.

ющий слияние двух глюонов:

$$
\frac{\partial N_{x y}}{\partial \tau}=\bar{\alpha}_{\mathrm{s}} \int \frac{d^{2} z_{\mathrm{t}}}{2 \pi} \frac{\left(x_{\mathrm{t}}-y_{\mathrm{t}}\right)^{2}}{\left(x_{\mathrm{t}}-z_{\mathrm{t}}\right)^{2}\left(y_{\mathrm{t}}-z_{\mathrm{t}}\right)^{2}}\left(N_{x z}+N_{z y}-N_{x y}-N_{x z} N_{z y}\right) .
$$

На рис. 4 представлено численное решение уравнения (5) $N_{\tau}\left(r_{\mathrm{t}}\right)$ при двух различных энергиях $\tau_{1}$ и $\tau_{2}$. Видно, что при высокой энергии (при малых $x$ ) величина $N_{\tau}$ растет, но остается меньше единицы, что разрешает проблему унитарности. Переход между малыми и большими $r_{\mathrm{t}}$ происходит на характерных значениях $r_{\mathrm{t}} \sim 1 / Q_{\mathrm{s}}(\tau)$, что разрешает проблему инфракрасной диффузии. Полученные результаты показывают, что излом решения происходит при меньших $r_{\mathrm{t}}$ (или при более высоких $Q_{\mathrm{s}}(\tau)$ ) для более высоких энергий $\left(\tau_{2}>\tau_{1}\right)$. Заметим, что $N$ мало при малых $r_{\mathrm{t}}$. Это явление называется цветовой прозрачностью, и оно оказывается не чем иным, как асимптотической свободой. Таким образом, решение уравнения Балитского-Ковчегова задает новый естественный масштаб $Q_{\mathrm{s}}$ теории КХД, представляющий собой импульс насыщения, зависящий от $s$ и $A$. Предполагая, что число частиц, рожденных в области центральных быстрот, пропорционально числу глюонов, произведенных в этой области, получим [8]

$$
\frac{d N}{d y} \sim x G_{\mathrm{A}}\left(x, Q_{\mathrm{s}}^{2}\right) \sim \frac{1}{\alpha_{\mathrm{s}}\left(Q_{\mathrm{s}}^{2}\right)} Q_{\mathrm{s}}^{2} S_{\mathrm{A}},
$$

где $S_{\mathrm{A}}$ - поперечное сечение взаимодействия, $S_{\mathrm{A}} \sim N_{\mathrm{A}}^{2 / 3}$ и $Q_{\mathrm{s}}^{2} \sim s^{\lambda} N_{\mathrm{A}}^{1 / 3}$. Тем самым, имеем

$$
\frac{1}{N_{\mathrm{A}}} \frac{d N}{d y} \sim \frac{1}{\alpha_{\mathrm{s}}\left(Q_{\mathrm{s}}^{2}\right)}
$$

и число частиц на одного участника взаимодействия оказывается не зависящим от числа этих участников по модулю $1 / \alpha_{\mathrm{s}}\left(Q_{\mathrm{s}}^{2}\right)$. Зависимость бегущей константы связи 


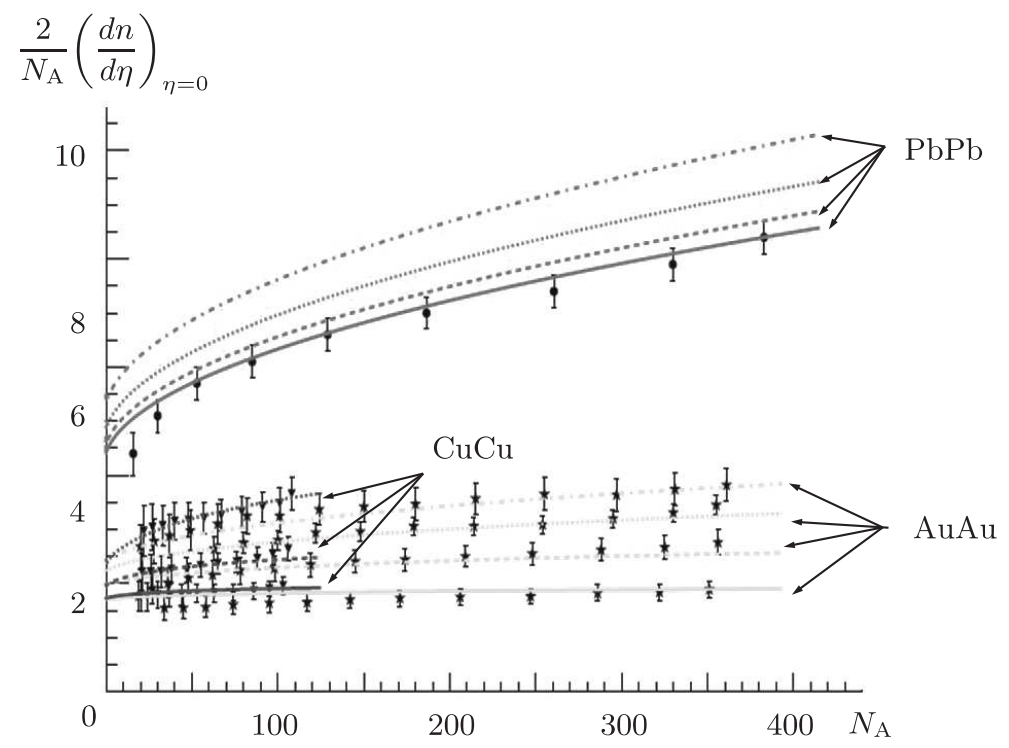

Рис. 5. Зависимость мультипликативности, отнесенной к $N_{\mathrm{A}}$, от числа участников $2 N_{\mathrm{A}}$ ). Кривые для $\mathrm{CuCu-соударений} \mathrm{(треугольники)} \mathrm{при} 22.4$, 62.4 и 200 ГэВ, АuАu-соударений (звезды) при 19.6, 62.4, 130 и 200 ГэВ и $\mathrm{PbPb-соударений} \mathrm{(кружки)} \mathrm{ри} \mathrm{2.76,} \mathrm{3.2,} 3.9$ и 5.5 ГэВ получены с помощью перколяций.

от $N_{\mathrm{A}}$ имеет логарифмический вид:

$$
\frac{1}{\alpha_{\mathrm{S}}\left(Q_{\mathrm{s}}^{2}\right)} \sim \ln \left(Q_{\mathrm{s}}^{2}\right) \sim \ln \left(s^{\lambda} N_{\mathrm{A}}^{1 / 3}\right),
$$

поэтому следует ожидать только логарифмический рост. Такое поведение находится в явном согласии с экспериментальными данными, как видно из зависимости на рис. 5, где показаны измеренные распределения мультипликативных быстрот для различных межъядерных соударений при энергиях БАК и RHIC как функции от числа участников. Эффект насыщения при этом четко прослеживается. Линии на рис. 5 отвечают описанию данных в подходе струнных перколяций [9], [10]. На рис. 6 показана энергетическая зависимость для центральных рр- и АА-соударений вместе с результатами, полученными с помощью струнных перколяций [11].

\section{4. ГЕОМЕТРИЧЕСКОЕ МАСШТАБИРОВАНИЕ}

Геометрическое масштабирование было предложено для описания глубоко неупругого рассеяния, в его основе лежит предположение о существовании масштаба типа насыщающего импульса $Q_{\mathrm{s}}[12]$. В соответствии с этим предположением поперечное сечение рассеяния виртуального фотона на протоне или поперечное сечение диполь-протонного рассеяния $\sigma_{\mathrm{dp}}\left(x, Q^{2}\right)$ зависят только от $Q^{2} / Q_{\mathrm{s}}^{2}(x)$. Это масштабирование довольно хорошо описывает глубоко неупругое рассеяние, и оно было распространено на рр-соударения [6], для которых данные в широком диапазоне энергий 0.9-7 ТэВ попадают на одну и ту же кривую [12], [13]. 


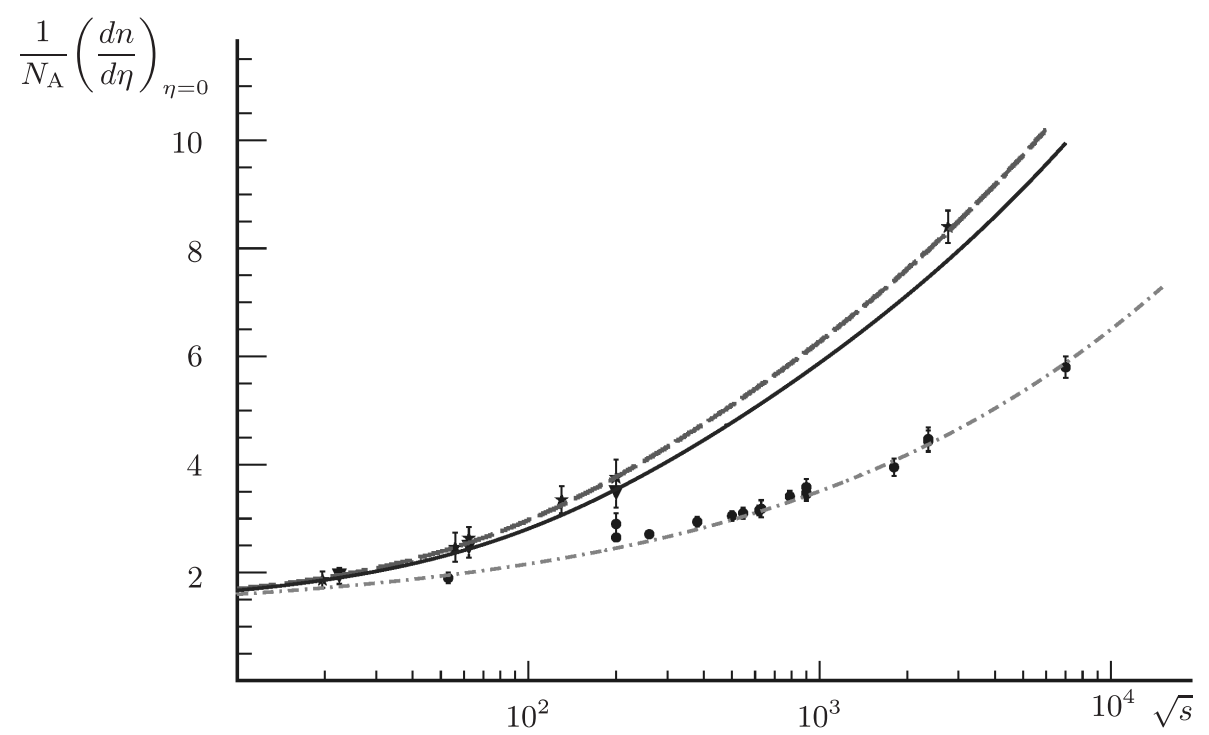

Рис. 6. Зависимость мультипликативности от $\sqrt{s}$. Кривые для рр (кружки), $\mathrm{CuCu}$ (треугольники), $\mathrm{AuAu}$ (звезды) и $\mathrm{Pb}-\mathrm{Pb}$ (звезды), полученные с помощью перколяций при $N_{\mathrm{A}}=A=1$ для рр-соударений (штрихпунктирная линия), при $N_{\mathrm{A}}=50, A=63$ для $\mathrm{CuCu}$-соударений (сплошная линия) и при $N_{\mathrm{A}}=175, A=200$ для $\mathrm{AuAu} / \mathrm{PbPb}$-соударений (штриховая линия).

В самом деле, в модели конденсата типа цветового стекла, инклюзивные поперечные сечения рассеяния зависят от $p_{\mathrm{t}}^{2} / Q_{\mathrm{s}}^{2}$ только при $p_{\mathrm{t}}<Q_{\mathrm{s}}$. Похожее масштабирование также получается в перколяции струн [14], [15]. Определяя насыщающий импульс в АА-соударениях $Q_{\mathrm{s}}^{\mathrm{A}}$,

$$
\left(Q_{\mathrm{s}}^{\mathrm{A}}\right)^{2}=\left(Q_{\mathrm{s}}^{\mathrm{p}}\right)^{2} N_{\mathrm{A}}^{\alpha(s) / 2} A^{1 / 6}\left(\frac{A}{N_{\mathrm{A}}}\right)^{1 / 3}
$$

в терминах рр-насыщающего импульса $Q_{\mathrm{s}}^{\mathrm{p}}$,

$$
\left(Q_{\mathrm{s}}^{\mathrm{p}}\right)^{2}=Q_{0}^{2}\left(\frac{W}{p_{\mathrm{t}}}\right)^{\lambda}
$$

где

$$
\alpha(s)=\frac{1}{3}\left(1-\frac{1}{1+\ln \left(\sqrt{s / s_{0}}+1\right)}\right)
$$

и $W=\sqrt{s} \cdot 10^{-3}, Q_{0}=1$ ГэВ, $\lambda=0.3$ и $\sqrt{s_{0}}=245$ ГэВ, можно показать, что для рр-, $\mathrm{AA}-$ и $\mathrm{pPb}$-соударений при всех энергиях и прицельных параметрах распределения поперечных импульсов зависят только от $\tau \equiv p_{\mathrm{t}}^{2} /\left(Q_{\mathrm{s}}^{\mathrm{A}}\right)^{2}$ при $\tau<1$ [16].

Рис. 7,8 демонстрируют, что это геометрическое масштабирование приблизительно удовлетворяется. 


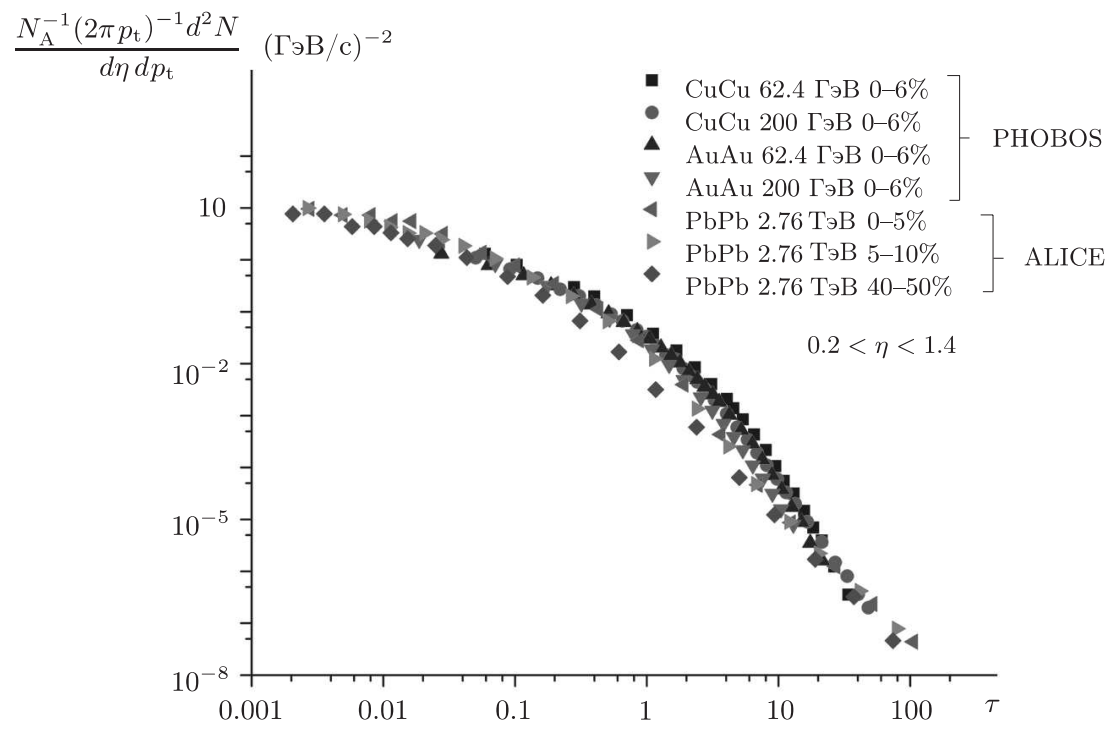

Рис. 7. Спектр поперечных импульсов как функция от $\tau$ при псевдобыстротах $0.2<\eta<1.4$ для центральных $\mathrm{AuAu}-$ и $\mathrm{CuCu}$-соударений при 62.4 и 200 ГэВ и для $\mathrm{PbPb-соударений} \mathrm{при} 2.76$ ТэВ.

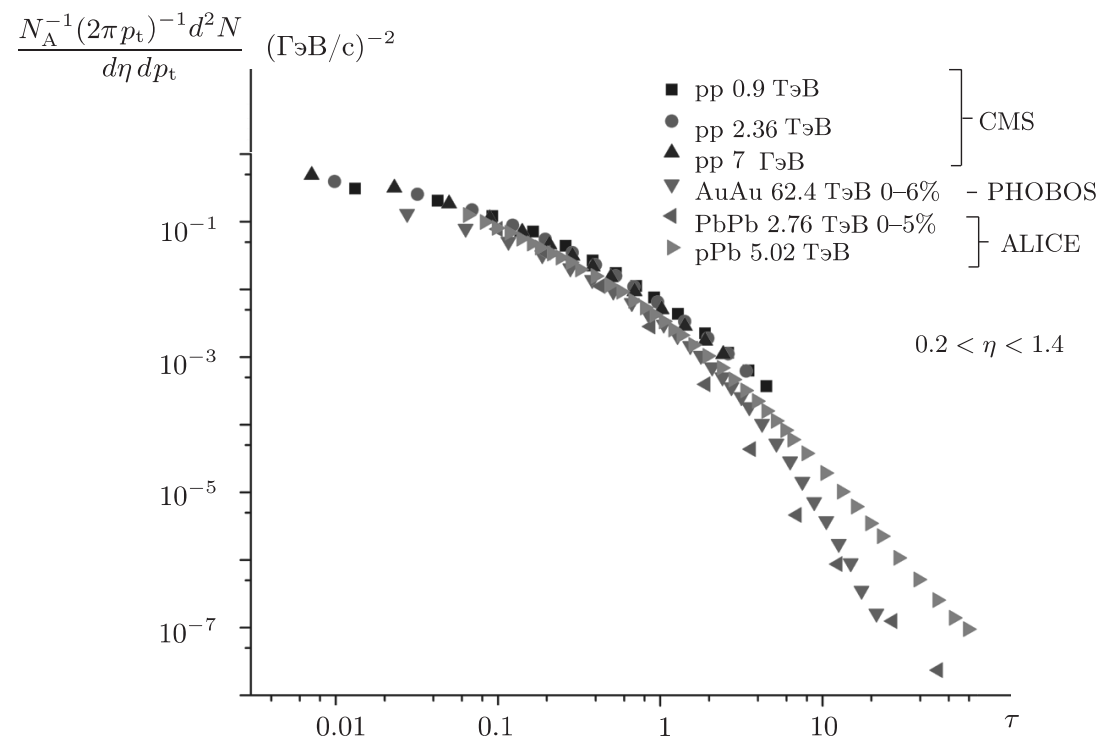

Рис. 8. Спектр поперечных импульсов как функция от $\tau$ при псевдобыстротах $0.2<\eta<1.4$ для рр-соударений, АuAu-соударений (центральность 0-6\%) при 62.4 ГэВ, $\mathrm{PbPb}$-соударений (центральность 0-5\%) при 2.76 ТэВ и pPb-coударений при 5.02 ТэВ. 


\section{5. ГАРМОНИЧЕСКИЙ ФУРЬЕ-АНАЛИЗ И КОЛЛЕКТИВНЫЙ ПОТОК}

Одно из наиболее интересных явлений, наблюдаемых в данных RHIC и БАK, это большой эллиптический поток $v_{2}$. Этот поток представляет собой вторую компоненту фурье-образа распределения поперечных импульсов произведенных частиц:

$$
\frac{d^{3} N}{d p_{\mathrm{t}} d y d \phi}=\frac{d^{2} N}{d p_{\mathrm{t}} d y}\left[1+\sum_{n=1}^{\infty} 2 v_{n}\left(p_{\mathrm{t}}\right) \cos \left(\phi-\phi_{0}\right)\right] .
$$

Ненулевой анизотропный поток $v_{2}$ существует в случае, когда измеряемые частицы в конечном состоянии зависят не только от локальных физических условий в точке рождения, но также и от глобальных геометрических свойств события. В релятивистской локальной теории такая нелокальная информация может проявляться только в форме коллективных явлений, возникающих благодаря взаимодействиям между некоторыми степенями свободы, локализованными в разделенных точках области соударения. В этом смысле анизотропный поток оказывается особенно отчетливым и сильным проявлением эффекта коллективной динамики в соударениях тяжелых ионов. Большое значение величины $v_{2}$ можно качественно объяснить следующим образом. При высокоэнергетических соударениях спектаторы передвигаются достаточно быстро для того, чтобы освободить путь, оставляя за собой на средней быстроте миндалевидную азимутально-несимметричную область материи КХД. Пространственная асимметрия предполагает наличие неравных градиентов давления в поперечной плоскости, с большим градиентом плотности в плоскости реакции (внутренней плоскости), перпендикулярной поперечной плоскости. Вследствие последующего многократного взаимодействия между степенями свободы эта пространственная асимметрия приводит к анизотропии в пространстве импульсов. Поперечный импульс испускаемой частицы скорее будет лежать во внутренней, а не во внешней плоскости, в силу чего имеем $v_{2}>0$.

Экспериментальные данные показывают, что эллиптический поток сначала возрастает с ростом $p_{\mathrm{t}}$, а затем выходит на постоянное значение вблизи $p_{\mathrm{t}} \sim 2$ ГэВ. Также видно, что зависимая от $p_{\mathrm{t}}$ величина $v_{2}$ идентифицированных адронов проявляет упорядочение по массам при низких значениях $p_{\mathrm{t}}$ и допускает примерный подсчет числа составляющих кварков. С другой стороны, величина $v_{2}$ нормирована на масштабе эксцентриситета на отношение между кратностью рожденных частиц и площадью перекрытия соударения. Большинство данных можно объяснить в рамках гидродинамической модели, указывающей на существование партонной среды с очень низкой сдвиговой вязкостью при отношении плотности энтропии, близком к предсказаниям $\mathrm{AdS} / \mathrm{CFT-соответствия} \eta / s=1 / 4 \pi$ [17]. Модель струнных перколяций также обеспечивает хорошее согласие с экспериментальными данными [18], [19]. В ее подходе отношение $\eta / s$ также оказывается малым [20], как видно из рис. 9. В этом подходе пространственная асимметрия преобразуется в асимметрию поперечного импульса из-за потери энергии произведенных частиц в кластере, вызванной взаимодействием с цветовым полем других струнных кластеров [21].

Важно не только исследовать величину $v_{2}$; старшие гармоники также помогают прояснить некоторые аспекты рассеяния, в частности явления вязкости в процессах рассеяния. 


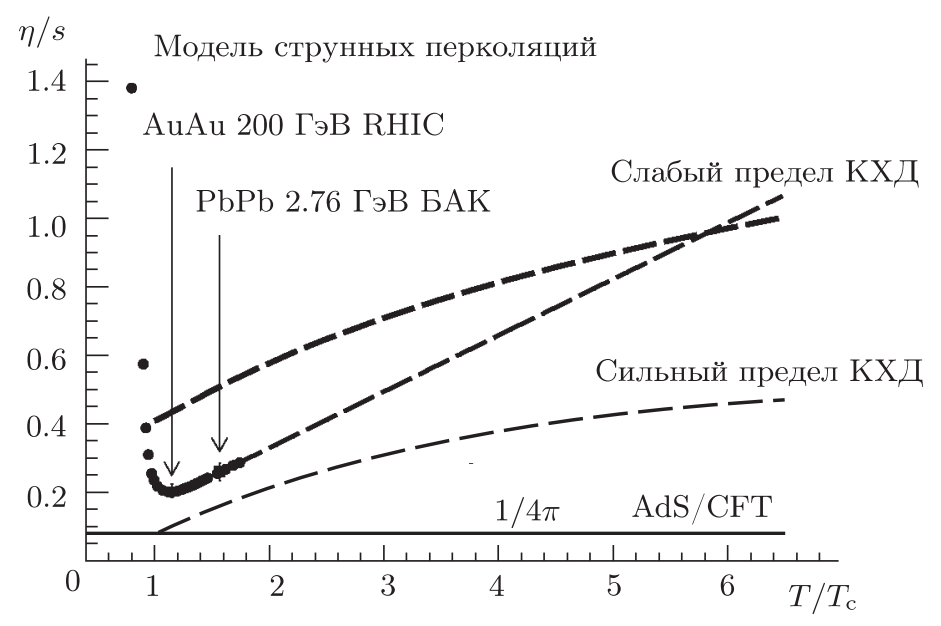

Рис. 9. Сдвиговая вязкость плотности энтропии как функция отношения $T / T_{\mathrm{c}}$ в подходе струнных перколяций. Также показаны предсказание на основе AdS/CFT-соответствия и ожидаемые кривые в сильном и слабом пределах КХД.

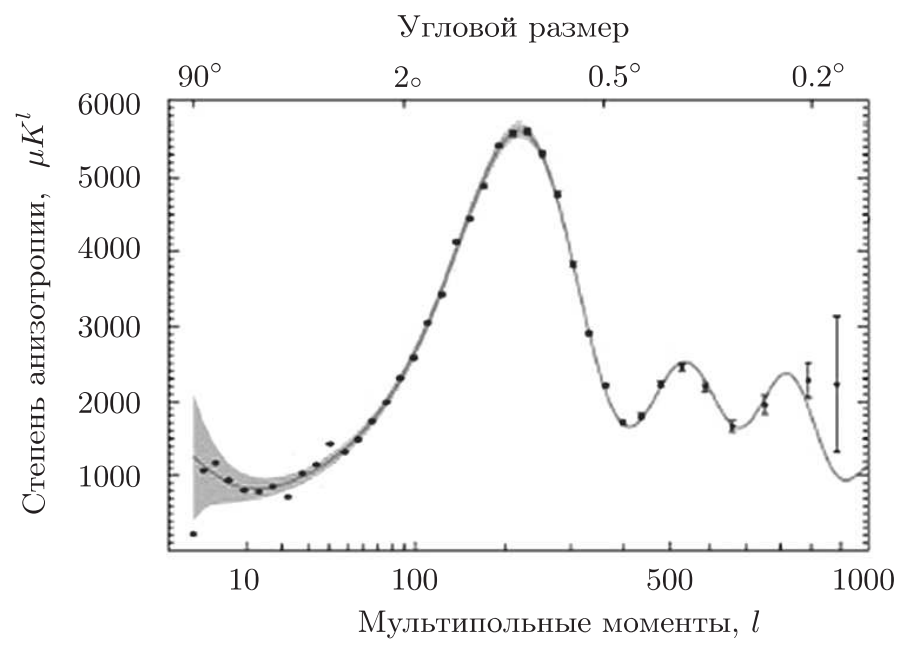

Рис. 10. Флуктуации температуры КМФ как функция от мультипольных моментов и углового размера.

Спектр величины $v_{n}^{2}$ при рассеянии тяжелых ионов можно сравнить со спектром мощности космического микроволнового фона (КМФ). Очевидно, что, в отличие от случая соударений тяжелых ионов, эффекты вязкости не играют существенной роли в ранней Вселенной в силу большого размера Вселенной по сравнению с характерным масштабом вязкости (длиной свободного пробега). На рис. 10 показаны флуктуации температуры КМФ в зависимости от мультипольных моментов $l$ и от углового размера. На рис. 11 величина $v_{n}$ представлена в виде функции от $n$, по- 


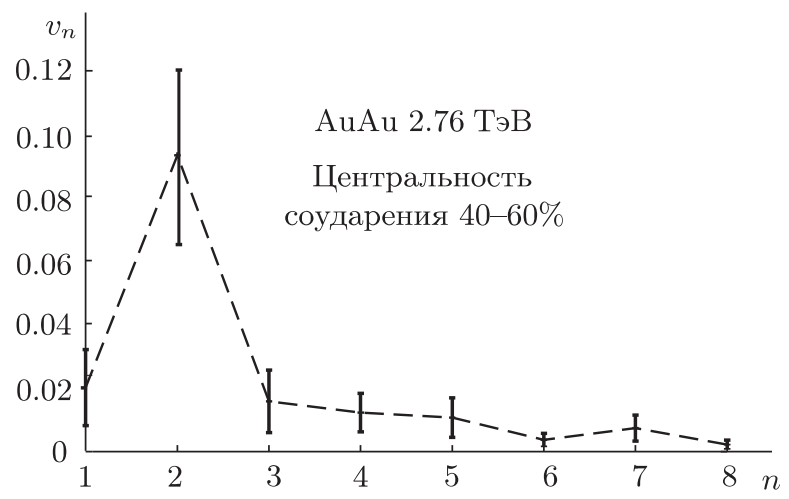

Рис. 11. Величины гармоник $v_{n}$, полученных в модели струн и струнных перколяций.

лученной в рамках подхода струнных перколяций. Видно хорошее совпадение с экспериментальными данными [22].

Видно, что спектр мощности КМФ продолжается до очень высоких импульсов, в то время как для спектра гармоник только первые 4-5 моментов дают заметный вклад. С другой стороны, спектр КМФ имеет максимум при $l \simeq 200$. Эти различия возникают из-за подавления высших гармоник эффектами типа вязкости и подавления низших моментов акустическим горизонтом.

\section{6. ДАЛЬНИЕ КОРРЕЛЯЦИИ БЫСТРОТ И ХРЕБТОВАЯ СТРУКТУРА}

Дальние корреляции быстрот дают информацию о ранних стадиях процесса соударения, а следовательно, и о начальном состоянии рассеяния.

Дальние корреляции быстрот измеряются с помощью отношения $b$ между дисперсией кратности рассеяния вперед-назад и дисперсией кратности рассеяния вперед (или назад):

$$
b=\frac{\left\langle n_{\mathrm{B}} n_{\mathrm{F}}\right\rangle-\left\langle n_{\mathrm{B}}\right\rangle\left\langle n_{\mathrm{F}}\right\rangle}{\left\langle n_{\mathrm{F}}^{2}\right\rangle-\left\langle n_{\mathrm{F}}\right\rangle^{2}} .
$$

Чтобы устранить близкие корреляции, между интервалами рассеяния вперед и назад введена большая запрещенная зона $\Delta y$.

Поведение этих дальних корреляций быстрот исследовалось экспериментально и теоретически. В подходе “конденсата цветового стекла" (КЦС) величина $b$ имеет следующее поведение [23]--[27]:

$$
b=\frac{1}{1+\alpha_{\mathrm{s}}^{2}\left(Q_{\mathrm{s}}^{2}\right) C} .
$$

Таким образом, $b$ возрастает с ростом энергии и центральности, поскольку уменьшается величина $\alpha_{\mathrm{s}}\left(Q_{\mathrm{s}}^{2}\right)$. Сходное поведение обнаруживается в подходе струнных 
перколяций. В этом случае имеем [22]

$$
b=\frac{1}{1+\left(1-e^{-\eta_{\mathrm{t}}}\right)^{-3 / 2}},
$$

где $\eta_{\mathrm{t}}$ - плотность струн, выраженная в единицах площади струн.

Взаимодействие между струнами также приводит к появлению корреляций между поперечными импульсами рассеяния вперед и назад и поперечным импульсом порождаемых частиц [28]-[34].

В дальних корреляциях быстрот проявляется хребтовая структура. Она впервые наблюдалась при энергиях RHIC в центральных $\mathrm{AuAu}-$ и $\mathrm{CuCu}$-соударениях, a впоследствии - при энергиях БАК в высокомультипликативных событиях $\mathrm{PbPb}$, $\mathrm{pPb}-$ и pр-соударений. В этих соударениях наблюдался коррелированный широкий максимум рождения частиц, простиравшийся на несколько единиц быстрот и локализованный вблизи азимутальных углов $\phi=0$ и $\phi=\pi$. Основные свойства хребровой структуры можно вкратце описать с помощью глазма-картины в подходе КЦС [25]-[27].

\section{7. ЗАКЛЮЧЕНИЕ}

Данные RHIC и БАК относительно рр- и АA-соударений выявляют интересные физические свойства, указывающие на то, что партонная среда при сильных взаимодействиях проявляет свойства жидкости с очень малой сдвиговой вязкостью для величины плотности энтропии. Сильное взаимодействие обеспечивается сильным цветовым полем, связывающим падающие частицы и мишень. Полевая конфигурация, предсказываемая в подходе цветовых трубок, или в глазма-картине, моделях КЦС или струнных кластеров, а также в подходе струнных перколяций, годится для описания дальних корреляций быстрот, распределений кратностей и распределений азимутальных гармоник.

Благодарности. Авторы благодарны организаторам IV Международной конференции "Модели квантовой теории поля" (MQFT - 2012) за подготовку прекрасной и интересной конференции. Работа поддержана Ministerio de Economía y Competitividad Испании (проект FPA 2011-22776), фондом FEDER, Spain Consolider CPAN Project и Правительством Галиции.

\section{Список литературы}

[1] ALICE Collaboration, Phys. Lett. B, 696:4 (2011), 328-337, arXiv: 1012.4035.

[2] L. V. Gribov, E. M. Levin, M. G. Ryskin, Phys. Rep., 100:1-2 (1983), 1-150.

[3] Y. V. Kovchegov, E. M. Levin, Quantum Chromodynamics at High Energy, Cambridge Univ. Press, Cambridge, 2012.

[4] V.S. Fadin, E. A. Kuraev, L. N. Lipatov, Phys. Lett. B, 60:1 (1975), 50-52; Э. A. Kypaeв, Л. Н. Липатов, В. С. Фадин, ЖЭТФ, 72:2 (1977), 377-389.

[5] Я. Я. Балицкий, Л.Н.Липатов, ЯФ, 28:6 (1978), 1597-1611.

[6] I. I. Balitsky, Nucl. Phys. B, 463:1 (1996), 99-157, arXiv: hep-ph/9509348.

[7] Yu. V. Kovchegov, Phys. Rev. D, 60:3 (1999), 034008, 8 pp., arXiv: hep-ph/9901281.

[8] D. Kharzeev, E. Levin, M. Nardi, Nucl. Phys. A, 747:2-4 (2005), 609-629, arXiv: hep-ph/0408050. 
[9] N. Armesto, M. A. Braun, E. G. Ferreiro, C. Pajares, Phys. Rev. Lett., 77:18 (1996), 3736-3738, arXiv: hep-ph/9607239.

[10] M. A. Braun, C. Pajares, Eur. Phys. J. C, 16:2 (2000), 349-359, arXiv: hep-ph/9907332.

[11] I. Bautista, J. G. Milhano, C. Pajares, J. Dias de Deus, Phys. Lett. B, 715:1-3 (2012), 230-233, arXiv: 1204.1457; I. Bautista, J. Dias de Deus, J. G. Milhano, C. Pajares, Phys. Rev. C, 86:3 (2012), 034909, 5 pp., arXiv: 1206.6737.

[12] L. McLerran, M. Praszalowicz, Acta Phys. Polon. B, 41:8 (2010), 1917-1926, arXiv: 1006.4293; Acta Phys. Polon. B, 42:1 (2011), 99-103, arXiv: 1011.3403.

[13] M. Praszalowicz, Phys. Rev. Lett., 106:14 (2011), 142002, 4 pp., arXiv: 1101.0585.

[14] J. Dias de Deus, E. G. Ferreiro, C. Pajares, R. Ugoccioni, Eur. Phys. J. C, 40:2 (2005), 229-241, arXiv: hep-ph/0304068.

[15] C. Pajares, Eur. Phys. J. C, 43:1-4 (2005), 9-14, arXiv: hep-ph/0501125.

[16] C. Andrés, A. Moscoso, C. Pajares, Nucl. Phys. A, 901 (2013), 14-21, arXiv: 1212.3102.

[17] P. K. Kovtun, D. T. Son, A. O. Starinets, Phys. Rev. Lett., 94:11 (2005), 111601, 4 pp., arXiv: hep-th/0405231.

[18] I. Bautista, J. Dias de Deus, C. Pajares, Eur. Phys. J. C, 72 (2012), 2038, 8 pp., arXiv: 1102.3837 .

[19] M. A. Braun, C. Pajares, Eur. Phys. J. C, 71 (2011), 1558, 9 pp., arXiv: 1008.0245.

[20] J. Dias de Deus, A. S. Hirsch, C. Pajares, R. P. Scharenberg, B. K. Srivastava, Eur. Phys. J. C, 72 (2012), 2123, 6 pp., arXiv: 1106.4271.

[21] M. A. Braun, C. Pajares, V.V. Vechernin, Nucl. Phys. A, 906 (2013), 14-27, arXiv: 1204.5829.

[22] J. Dias de Deus, C. Pajares, Phys. Lett. B, 695:1-4 (2011), 211-213, arXiv: 1011.1099.

[23] N. Armesto, L. McLerran, C. Pajares, Nucl. Phys. A, 781:1-2 (2007), 201-208, arXiv: hep-ph/0607345.

[24] N. Armesto, M. A. Braun, C. Pajares, Phys. Rev. C, 75:5 (2007), 054902, 8 pp., arXiv: hep-ph/0702216.

[25] S. Gavin, L. McLerran, G. Moschelli, Phys. Rev. C, 79:5 (2009), 051902, 4 pp., arXiv: 0806.4718.

[26] F. Gelis, T. Lappi, R. Venugopalan, Phys. Rev. D, 79:9 (2009), 094017, 7 pp., arXiv: 0810.4829.

[27] Y. V. Kovchegov, D. E. Wertepny, Nucl. Phys. A, 906 (2013), 50-83, arXiv: 1212.1195.

[28] M. A. Braun, C. Pajares, Phys. Rev. Lett., 85:23 (2000), 4864-4867, arXiv: hep-ph/0007201.

[29] M. A. Braun, C. Pajares, V. V. Vechernin, Phys. Lett. B, 493:1-2 (2000), 54-64, arXiv: hep-ph/0007241.

[30] M. A. Braun, R. S. Kolevatov, C. Pajares, V. V. Vechernin, Eur. Phys. J. C, 32:4 (2004), 535-546, arXiv: hep-ph/0307056.

[31] V. Kovalenko, V. Vechernin, Model of pp and AA collisions for the description of long-range correlations, arXiv: 1212.2590 .

[32] V. Vechernin, Correlations between multiplicities in windows separated in azimuth and rapidity, arXiv: 1210.7588 .

[33] N. Armesto, D. A. Derkach, G. A. Feofilov, Phys. At. Nucl., 71:12 (2008), 2087-2095.

[34] V. Vechernin, I. Lakomov, PoS (Baldin ISHEPP XXI), 2012, 072, 12 pp., arXiv: 1212.2667. 